\title{
Design and evaluate intelligent control safety systems on the TOMI robot
}

by Zhao, C., Blackmore, S., Warbrick, M. and Wane, S.

Copyright, Publisher and Additional Information: This is the authors' accepted manuscript. The final published version (version of record) is available online via Taylor \& Francis.

Please refer to any applicable terms of use of the publisher.

DOI: $\underline{\text { https://doi.org/10.1007/s10586-018-2228-6 }}$

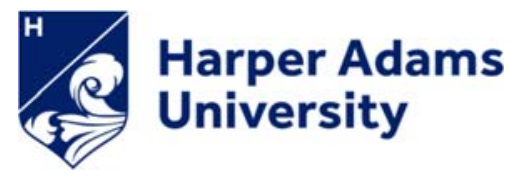

Zhao, C., Blackmore, S., Warbrick, M. and Wane, S. 2018. Design and evaluate intelligent control safety systems on the TOMI robot. Cluster Computing. 


\section{Design and Evaluate Intelligent Control safety systems on the TOMI Robot}

\author{
Chunhua, Zhao \\ Gansu Agricultural University; \\ NO.1.Yin Men Country. Anning District, \\ Lanzhou city, Gansu Province, 730070, China \\ E-mail: zhaoch@gsau.edu.cn \\ Simon Blackmore, \\ Harper Adams University; \\ Newport, Shropshire, \\ TF10 8NB, Great Britain \\ E-mail:simon.blackmore@harper-adams.ac.uk
}

\author{
Michael Warbrick \\ Harper Adams University \\ Newport, Shropshire, \\ TF10 8NB, Great Britain. \\ E-mail: mwarbrick@harper-adams.ac.uk \\ Sam Wane \\ Harper Adams University \\ Newport, Shropshire, \\ TF10 8NB, Great Britain \\ E-mail: swane@,harper-adams.ac.uk
}

\begin{abstract}
Aimed to design safety systems and evaluate the behaviours about Robot grass cutting named TOMI, the False Tree Analysis, Failure Mode Effects Tree Analysis methods were used for review and analysis about the TOMI Robot, it would be liability and legislation; TOMI Robot management embedded guidelines and knowledge such as Agricultural Engineering, Design and manufacture of agricultural machinery, Mechanic Theory, Mathematics, Electronics, Grass science, Computer science and several software programs; Procedure and Reliability analysis for robots TOMI safety
\end{abstract} systems are key features, the safety systems of Agricultural Robot such as TOMI should be checked in various working circumstance; With the full consideration of engineering practicability, the solutions to the safety problems of the TOMI robot are promoted, Technology Route and models about TOMI's safety system were built, Process Management 、 continual improvement tools and Techniques and effects analysis were built in the new safety systems of TOMI Robot.TOMI Function Measurements such as Braking, throttle and pedal force were tested and analysised.TOMI's Mechanical system 、Hydraulics and Electrical Systems were tested for checking safety and evaluated, some sensors and laser such as Distance sensors, SICK, GPS, Dead man handle, safety red button and bumpers were built up and developed the TOMI Robot's new safety systems; To ensure the safety and reliable operation is a system engineering, it is involved to various TOMI robot design, production, operation, adjust, and management; to improve the TOMI robot reliability and reduce the failure frequency was an important way to improve the robot inherent safety; The Evaluation Criteria of
Robot grass Cutting DFMEA occurrence may be suggested to use multiple complex technology knowledge and design with more experience. Application built with Microsoft Robot Development studio was run over on the www.webfarming.com. The hazard and risk analysis were detailed about the safety problems of TOMI robot and deeply studied. Development more practical and safety TOMI Robot would be carried out at northwest China in the future.

Key words: TOMI Robot Grass cutting; intelligent control safety systems; Design and evaluate

\section{Introduction}

With the rapid development of agricultural robot technology, the safety level of robots has become very important for enhancing robots in application. The safety system was designed to be used with whole-region coverage intelligent TOMI robot. The development of safety system used in agricultural robotics was slow except a few type of mower robots have been studied for cutting lawn growing in ZheJiang university without planing proper path routing or control systems disorder;several mower robots were run at random in the lawn, an early publication was named "Robot reliability, maintainability, safety" (Liao, 1994); however since then, few perfect papers about robot safety systems have been published in China. The research about TOMI robot's safety systems using for cutting forages were specifically reviewed basing on three hazard analysis techniques: FTA, FMETA and HATAP methods ${ }^{[1-2]}$. Technology Route and models about TOMI's new safety system were built and designed; the hazard and risk analysis are detailed and evaluated to solve the new development safety problems about TOMI robot's grass cutting.

Receiver Date: 2-2-2018:

The Fund projects of supporting: National natural fund projects; the Ministry of Agriculture Utilities Industrv (51565002); Special Fund for Agro-scientific Research in the Public Interest (201203024); Research and Development of the main Project in Gansu province (17YFINA061) and The First Fuxin's outstanding talent Candidates project in Gansu Agricultural University

Biographies: Chunhua Zhao (1972-), female, Ph.D, professor, major in agricultural mechanization engineering and biomechanical,. E-mail: zhaoch@gsau.edu.cn. Lanzhou. Gansu, 730070

Correspondence author: Simon Blackmore(1954-), the leader of Engineering College, Harper Adams University;

E-mail: simon.blackmore@harper-adams.ac.uk 


\section{Review on safety systems used for Robotic grass cutting}

It is well known that the development of safety systems used for robot grass cutting need to be designed, the Robot was named smart TOMI, generally, there are seven levels of safety steps may be followed: 1.Route planning; 2 . Laser range finder; 3.Dead man's handle;4. Wide area safety curtain; 5.Collapsible bumper;6. Big Red buttons; 7. Small, Slow and light by design. These may include their integration with Agricultural Robots; liability or legislation; safety to person v crops and itself; Robustness, Robot management were embedded guidelines and knowledge. As a project to carry out, a research group was needed, the research group need to be familiar with many fields of knowledge such as Agricultural Engineering, Design and manufacture of Agricultural machinery, Mechanic Theory, Mathematics, Electronics and Electronics Engineering control systems, Grass science, Computer science and several software programs used for Robot TOMI. it shows that the following detail contents:

A. Procedure analysis for robots TOMI safety systems: For a workplace to be safe, the careful implementation of safety measure is necessary, the safety systems of TOMI robots should be checked by every operators in various working circumstance; the procedure of safety systems is that TOMI robot should be safety to person; then, it should be safety to crops, and last, it should be safety to itself;

B. Relationships between Fault Trees and RBDs: its most valuable result is the collective technology of cross-functional teams. Part of the evaluation and analysis is the assessment of TOMI's risk. The important point is that a discussion conducted regarding the design (product or process), review of the TOMI robot's functions and any changes in application, and the resulting risk of potential failure. The Advanced Product Quality Planning (APQP) process identifies several general areas of focus in the process on TOMI safety system development; C.TOMI's team leader who has carried out TOMI safety systems have several means of assuring that recommended actions are implemented. they include the following actions: a. Reviewing the present devices designs and processes about new safety systems of TOMI Robot, b. Confirming the incorporation of changes to design and assembly manufacturing according the advanced technology and documentation, c. Reviewing Design and Process with FMEAS method, special FMEA applications, and Control Plans;

D. Overview of FMEA Strategy: Planning and Implementation of TOMI's technology;

E. DFMEA Design Failure Mode and Effects Analysis about TOMI: a. A team of TOMI Robot with responsible leader who has experience in assembly, manufacturing, design, analysis, test, reliability, materials, quality, service, and suppliers for the next higher assembly system and component about robot ${ }^{[3-4]}$.TOMI is expected to directly and actively involved in all affected areas; $b$. Manufacturing, Assembly and Service ability were Considerably; c. Parameter Diagrams. The function of TOMI is useful in identifying static and dynamic states correspond to the Potential Failure Modes in the DFMEA; d. Body of the DFMEA Form: The body of FMEA Contains the analysis of risks was related to the potential failures, and improvement action being taken; e. In the development of the FMEA, The identification of all potential causes of the failure mode is key to subsequent analysis to TOMI's safety.

F. Process Failure Mode and Effects Analysis about TOMI: The PFMEA does not rely on product design changes to overcome limitations in the process, the team ensure that the risk is addressed through existing design controls or recommended action as documented in the FMEA such as Risk Evaluation; Risk Priority Number: one approach to assist in action prioritization has been to use the Risk Priority Number: RPN $=$ Severity $(\mathrm{s}) \times$ Occurrence $(\mathrm{O})$ $\times$ Detection(D); If the environment is composed of high humidity and subsystems, interactioning among non-contacting subsystems can be relatively difficult to predict but important. The safety systems of Tomi robot should be considered analysis, testing, reviews, and development. The improvement Guide such as figure 1 shows the following contents: The Safety of TOMI Robot is a Process Management, it is not only need continual improve tools and Techniques, but also should Statistical Process Control; FMEA method was built in Safety control systems of TOMI Robot which were used "Programming Microsoft Robotics Studios"(Sara, 2008). Technical and safety requirements were built in Safety systems of TOMI Robot: The American National Institute of Standards and Technology (NIST) has identified three safety zones for working robots (Kilmer, 1982): with Level 1 safety zone was defined as the area outside the accessible working area of the robot; Level 2 zone as the accessible workspace area of the robot (excluding a small area surrounding the robot itself) 
and Level 3 as the zone in the immediate vicinity of the robot. Fig. 1 depicts the concept of dividing the space around the TOMI Robot into the respective safety zones. Apart from maintaining a safe distance for humans, further measures should still be considered for the safe use of agriculture robots.
However, in the cases of domestic agriculture robots use, keeping humans far from robots appears to be impossible. Thus, it is clear that safety of humans under conditions of close proximity should be given proper consideration, especially smart agriculture robots would be adapted for using in mountain area.

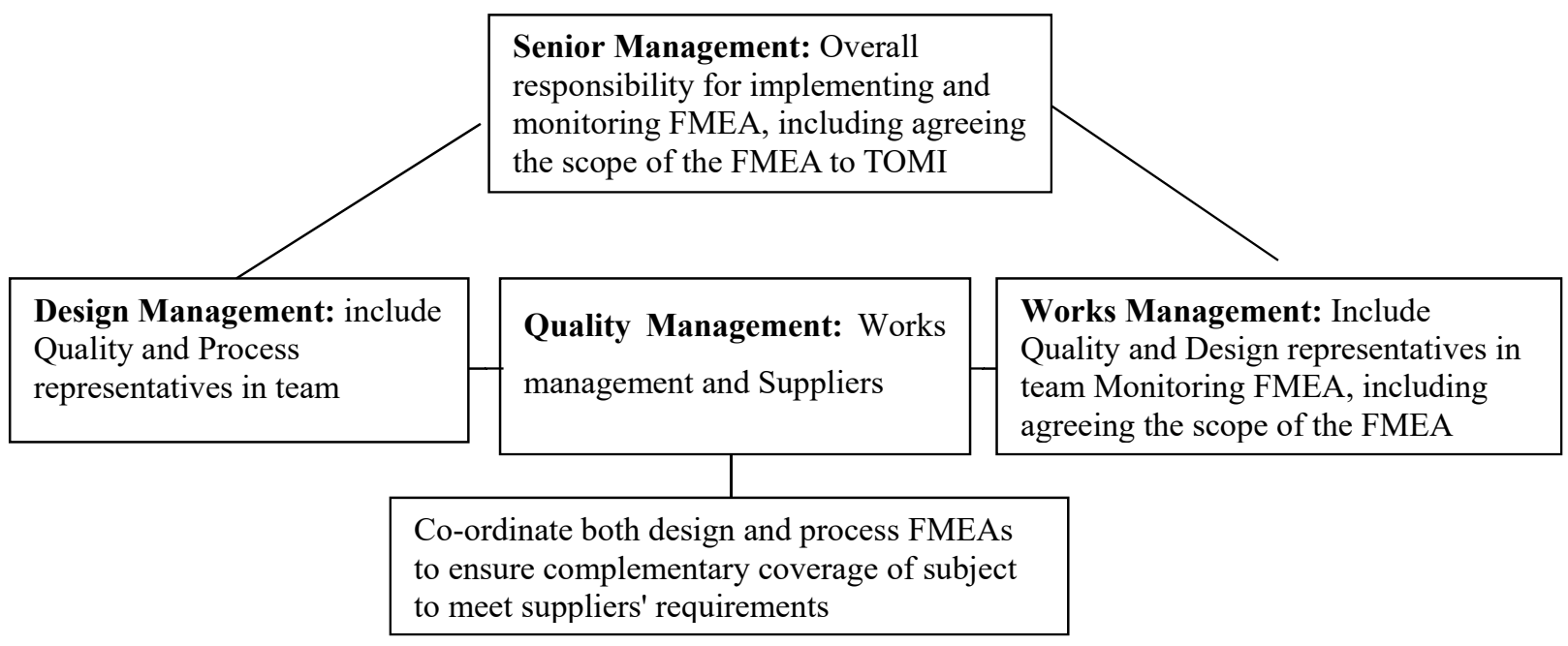

Figure 1.The Diagram of TOMI Robot Structure for FMEA Management

The autonomous safety system implement carriers were navigated in a deterministic manner within the Autonomous Hectares, Based on a predefined route plan with embedded actions, developed and supplied. The visualization and verification of the predetermined rout plan would be simulated or visualized in real time with software Architecture for TOMI Robots. To realize the aim, the safety system can accurate accepting dates or information automatically from different devises sensors about static or moving obstacles, and implement status and performance in all tasks carried out in the field. all the optical and economical details for cutting speed ratios would be collected, the reel speed ratios, control the accurate time of stop by Deadman handle when facing up to special circumstance and the fields working technology data also would be collected. To enhance the system's controlling flexibility and extensibility and help farmers more economical in their tasks. The details would be analysed and used to benefit farm management decisions and improve efficiency. The safety system will monitor every movement made by the farmer. The safety system will also hold all the details about which the vehicle and implements used and tell the operator which ones are best for particular jobs.

Following the steps, some of best technology assets will be invested such as Tomi's key Components such as GPS systems and Trimble systems connected with Microsoft Robotics Studio about development of new Tomi Robotics. MSRS contains services and tools that allow engineers to design, develop them robotics ${ }^{[3-5]}$.Application built with MSRS will run over on the WebFarming internet, the platform will be built by developers on Web-farming robotics applications ${ }^{[6-8]}$. it has been difficult for new programmers and engineers to enter the field of robotics, but MSRS offers a standard platform that can be used by everyone just as office management software system.

Safety systems of TOMI Robot emphasize on the need for an innovative certification procedure for TOMI robots with the view to test the validity of the applied measures and to also provide supplementary guidelines to the existing standards and methods. It would be well-established standards development not only research in precision Agriculture Engineering but also build in international cooperation platform. 


\section{Evaluate existing safety system on the TOMI robot}

Safety systems of TOMI Robot can produce safely Agricultural Robots for human, no matter what failure, malfunction or mishandle may occur.

The TOMI's system function, structure ,

principles and test TOMI robot's safety systems were studied and analyzed in the Harper Adams University, a few Structural parts which have been designed in TOMI would be evaluated its safety, for example, Failure Mode of Return spring fitted in reverse making pedal does not return, it is easy to operate error and unsafely; a few sensors and switches "have been designed "electrical components in TOMI will be evaluated its safety, more information and research methods ${ }^{[1,4,15]}$ are available from previous similar designs and research experience.

The safety systems of TOMI robotic grass cutting are consisted of Mechanical systems, Hydraulic systems and Electronic systems such as fig2. Using all kinds of sensors can enhance safety system's controlling flexibility and extensibility, Communication and protocol implement sensors such as RS2100 distance sensor can scan 11 segments with totally $88^{\circ}$ degree in $3 \mathrm{D}$ directions detecting various obstacles, Diameter of the light spot is up to $550 \mathrm{~mm}$ at $4 \mathrm{~m}$ (from central to beam) ${ }^{[15-}$ ${ }^{17]}$, and testing results showed that it can scan person with $0.30 \mathrm{~m} \times 0.15 \mathrm{~m} \times 1.65 \mathrm{~m}$ high about $0.2-10 \mathrm{~m}$ distance; and SICK Laster LMS511scanner can detect $190^{\circ}$ scanning beam with a unit, accessories such as RS232、LED and Relay were designed to connected computer through TOMI'S electronic control safety system between the hardware systems and software systems. It is ensured that the modified safety systems implements can be coupled directly to TOMI robot. The most distinctly implement is needed for cutting the grass with high effective and safety. The safety systems of technology development was followed the specifications resulting from the used-driven design. TOMI should be instrumented appropriately to detect collisions to solid objects in the field; the safety systems must reflect it very sensitive and precise. "Development of safety systems used for robotic grass cutting"was researched and developed in intelligent control system. The original Safety system of TOMI robot have been included the following items:

\subsection{Mechanical Safety system about TOMI Robot}

1. The distance between the left clutch Pedal and Brake pedal of mechanical structure is only $322 \mathrm{~mm}$ long, that make the TOMI working in the field unsafely;

2. The structure of adjusting cut height is safety and convenient to TOMI Robot in field.

3. The safety protection structure of cutter is reliable in complex travelling conditions, and the Rear reel with double safety structure is more safety than the former two cutter reel in travelling.

4. Only three tyre of original TOMI is not perfect safety using for developing new TOMI Robot's safety system.

\subsection{Electric Safety system about TOMI Robot:}

1. Several safety electronic switches were checked such as seat switch , three reel switches、 Park Brake switch、Transaxle or neutral switch、 clutch switch;

2. Safety system of steering system was studied and tested; it is only safety on good condition.

3. Safety system of speeding system: Test showed that working safety speed should be adjusted to $2 \mathrm{~km} / \mathrm{h}$ when the condition of grass long or heavy grass or wet circumstance.

\subsection{Hydraulic safety system about TOMI Robot:}

Petrol engine on the TOMI Robot may be developed more safely in grassland, but unsafely using in mountain area. The TOMI robot may be safe to operate in dynamic environments which are in close proximity to humans, in order to achieve this safely, its hardware systems and software systems should be properly integrated. Software reliability is essential to make TOMI robot working flexibility and adaptability, and a complicated computer-based safety-critical system is essential to TOMI, it can avoid too dangerous actions happening with catastrophic consequences. It is important, therefore, to be able to recognize and identify potentially hazardous conditions in the operation of a TOMI robot at an early stage. Defining the procedure to determine the circumstances under which the robot may cause injuries and performing a hazard analysis is essential. Safety test methods include a system simulation following a risk analysis such as FTA, risk tree, network logic, preliminary hazard, energy barrier, task analysis, subsystem analysis, failure mode and effects analysis. These quantitative techniques make TOMI Robot use of probability theory to estimate relevant hazards. Moreover, a stochastic process based on probability theory could be implemented to a certain degree in the field of internal safety devices.

The IRM of TOMI system's function, structure, principles, the state of failure, the factors of failure were used by the FTA method to research the grass cutting, the research method is not only an innovation for the safety of TOMI robot, but also 
another innovation research ideas for the safety system of other agriculture robot. This method also accounts for the effects of thermal cycling on the component failure rate due to variations in the ambient temperature and component switch on and off. Security is an important performance index of TOMI robot. The TOMI robot safety system's main safety technology parameters such as the working stubble, the re-cutting rate, the grass loss rate and so on were tested in the field experiment at Harper Adams University. Applied comprehensive scoring method to obtain the optimal combination of working parameters, Measured with the optimal combination safety system of operational parameters, which could meet the demand of the smart forage harvester safely operation. To improve the safety of the TOMI Robot, to ensure the safe and reliable operation is a systems engineering, all of that needs various TOMI robot design, production, operation, adjust, management and other personnel involved be guaranteed at each stage the life cycle of the robot to take corresponding measures. At the same time, the engineering safety and reliability design of the Robot, Robot repair technology process has a close relationship. On one hand, in view of safety accidents occurred mostly in the TOMI robot failure, therefore, improve the TOMI robot reliability, reduce the failure frequency is an important way to improve the robot inherent safety; on the other hand, the effective check method, especially the preventive checks method to prevent TOMI faults is an important means to ensure the safe and reliable operation of the Robot, the elimination of major accident hazards cause TOMI robot safety accidents are mainly: (1) operating error; (2) Personnel abnormal intrusion; (3) Robot fault; Among them, the Operator operate errors and personnel into the factors leading to abnormal accident to person, and the Robot fault is the cause of the accident of the Robot itself, reflects the level of inherent safety robot. To test and analysis the TOMI robot in the field.The use of systematic techniques such as Fault and Event Tree analysis to examine the safety and reliability of a given robotic system were used in the safety systems of Tomi. The context of humanrobotic systems, an intervention is not only driven by component failures, but includes many other factors that can make the TOMI robotic agent to request or a Human agent to provide intervention. The effect of unplanned interventions on the effectiveness of human-robot systems were investigated analytically using traditional reliability analysis. The implications of these analytical trends were discussed on the design and evaluation of humanTOMI robot systems.

\section{Testing and Analysis the safety of Existing TOMI}

According former researching and referencing other scientist's research, a little valuable information showed that higher risks require higher integrity levels to ensure the performance of the safety function. Machine safety systems can be categorized as to their design intent and the ability to ensure the performance of the safety function. TOMI, originally as a semi-automatic cutting grass machine based on a small TOMI was used by many areas in UK. It was adapted to be person driving and had few safety systems installed. Development the safety system of TOMI need adapted to be driverless and have numerous safety systems. Before deciding upon building on new safety system, it is important to analysis the existing TOMI's structure, specification and working's Feature, aimed to seeing how and where to improve and develop the safety system to make TOMI working more effective and safety. There are many mechanical structures setting on the TOMI's robot, the braking tests, throttle force, brake force and pedal force were tested to analysis the safety of mechanical system as showing the following table 1:

\subsection{Braking Tests}

Table 1: Results of Braking Tests

\begin{tabular}{|c|c|}
\hline $\begin{array}{l}\text { Stopping } \\
\text { Distance }(\mathrm{mm})\end{array}$ & Description \\
\hline $\begin{array}{r}210 \\
178 \\
138 \\
\end{array}$ & $\begin{array}{l}\text { Full throttle just letting off forwards pedal(Three times safety test for checking the } \\
\text { mechanistic structure were repeated) }\end{array}$ \\
\hline 325 & $\begin{array}{l}\text { Full throttle, just getting up off seat, letting safety switch stop the engine (only once } \\
\text { can accurate test the Brak's sensitivity in such specific factors) }\end{array}$ \\
\hline $\begin{array}{c}159 \\
83 \\
105 \\
\end{array}$ & $\begin{array}{l}\text { Full throttle, letting off forwards pedal w/o pedal damper(Three times safety test the } \\
\text { Brak's sensitivity were repeated in such specific factors) }\end{array}$ \\
\hline 117 & $\begin{array}{l}\text { Idle throttle just letting off forwards pedal (one times can accurate test the } \\
\text { Brak's sensitivity in such specific factors) }\end{array}$ \\
\hline
\end{tabular}



such specific factors)

\section{2 Throttle Force}

The force required to move the throttle lever from idle to fully open at the carburettor was found to be $75 \mathrm{~N}$, however most of this resistance was found to be caused by the return spring. $60 \mathrm{~mm}$ of movement from chock point to Fast point was required to attain full throttle.

The test results showed that it was great

\section{Design new safety systems and behaviours about TOMI Robot.}

According to the human-robot system failure is analyzed, new safety systems and behaviours about robot TOMI have been designed and developed. Using the method of FTA analyze the potential fail factors of the intelligent robot TOMI, sum up the main problems impacting the safety of the robot TOMI and put forward some solutions for the safety problems of the robot TOMI. The appropriate difference stopping distances about testing the Brakes and pedals in the same throttle situation, after several repeat testing and checking, the nearest distances between the left clutch pedal and right brake pedal of the mechanical structure is only $322 \mathrm{~mm}$, it is so narrow distance structure that make the testing velocities was changed every time.

safety measures are compared in terms of their cost-effectiveness. Finally, ergonomics points of view are provided to prevent accidents due to the robot TOMI.TOMI'S Mechanical system and Electrical Systems were analysis, as well as consultation with Professor Simon Blackmore and other staffs, some sensors and laser such as RS2100 Distance sensors, SICK, GPS, Trimble, Laptop (Safar), Deadman Handle, Big Red button and Bumpers were built to develop the new safety system about TOMI robot grass cutting such as figure 2 .

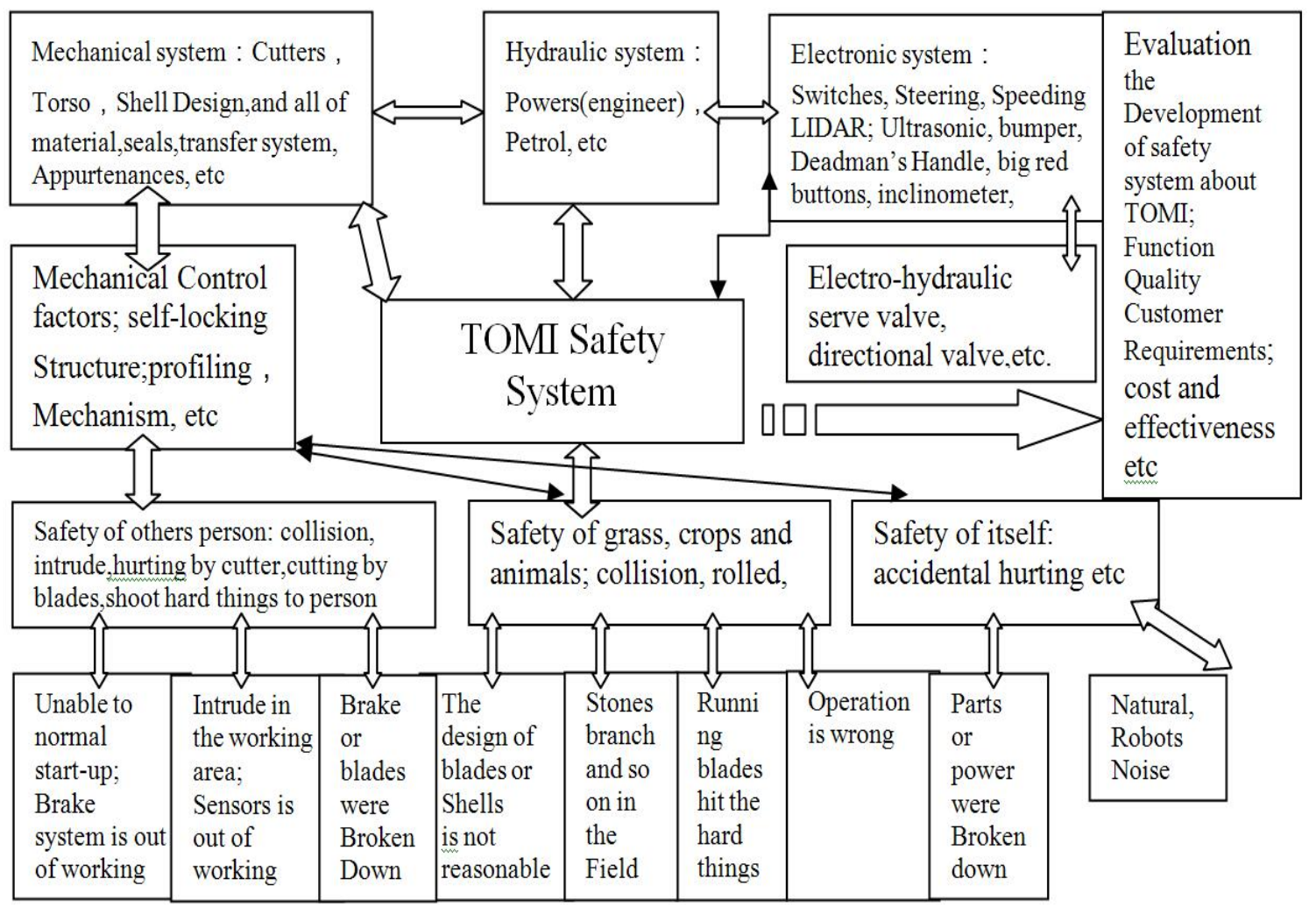

Figure2. The safety system about TOMI Robot scheme 


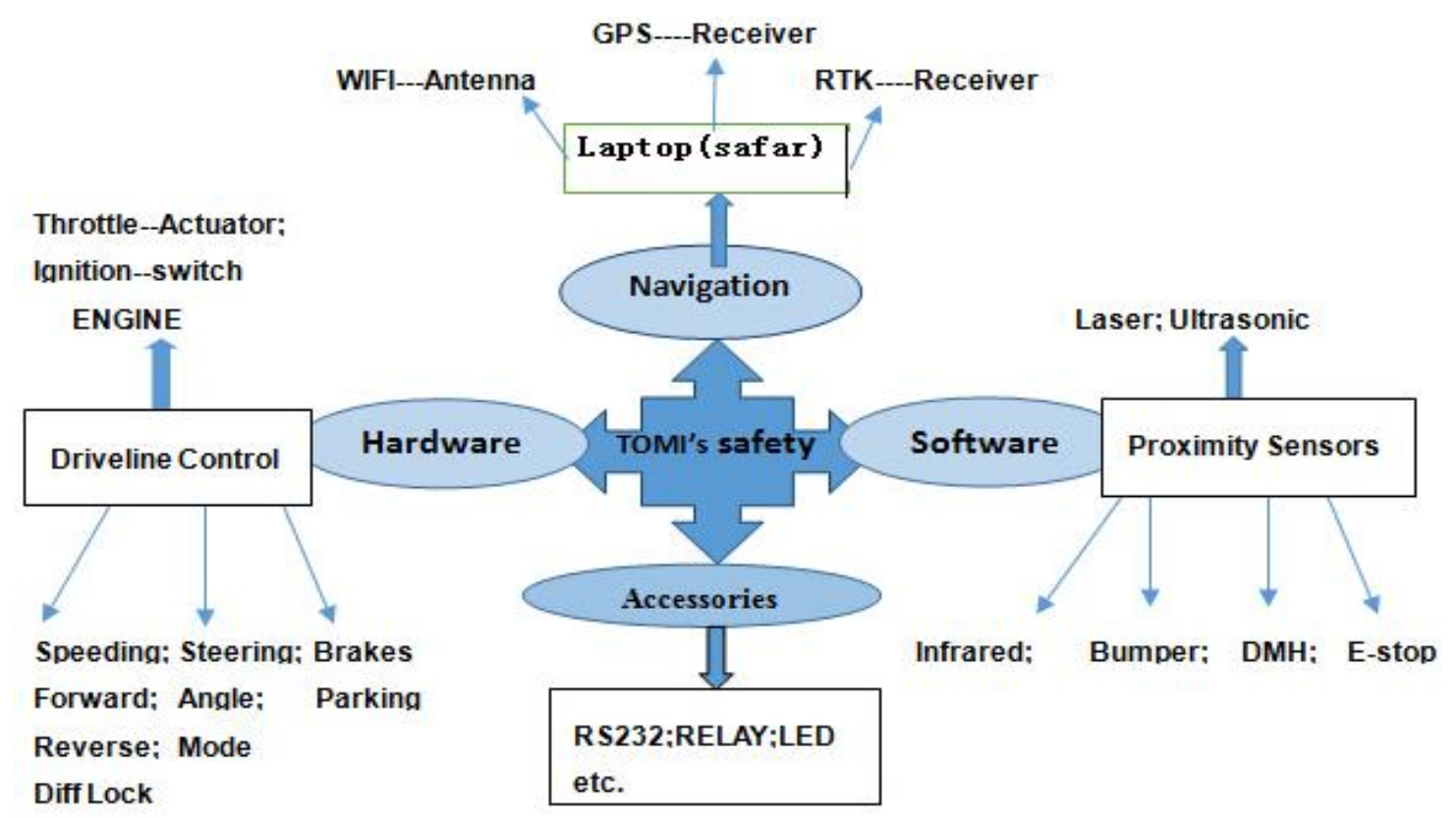

Figure3. Technology Route and model about TOMI's safety system

\subsection{Solving the safety system scheme about TOMI Robot}

As many ideas and associated parameters as possible to solve the safety systems about TOMI robot can be considered and were listed such as fig2. This provided scheme by which robot TOMI could be evaluated. According to the former research and analysis, following figure 2 about the technology route and model of Robot TOMI's safety system were built and developing; suitable accessories can be found at www.pepperl-fuchs.com. ${ }^{\text {9-13] }}$

Combined with fault tree analysis method of electromechanical system (FTA), fault tree analysis, based on the Intelligent Robot TOMI, the topics with discussing potential risk and designing the Intelligent TOMI Robot in Agricultural Engineering are the main safety problems, and the paper has been put forward the primary problem solving scheme such as fig2.

\subsection{Design requirements and protective measures}

System, technical and safety requirements were carried out to TOMI Robot: The American National Institute of Standards and Technology (NIST) has identified three safety zones for working Robots ${ }^{[20]}$, with Level 1 safety zone defined as the area outside the accessible working area of the Robot, Level 2 zone as the accessible workspace area of the Robot (excluding a small area surrounding the robot itself) and Level 3 as the zone in the immediate vicinity of the Robot. Fig2 and fig3 were showed TOMI'S safety scheme and systems.

Safety system requirements of relevant identified robot involved with the implementation and operation of automation technology about TOMI have been collected through direct testing. The acquired adaptable power and user requirements would be categorized and sorted as a preliminary step for a more formal safety system analysis. The Quality Function Deployment analysis would involve the ranking of technical specifications in relation to their degree of contribution to the fulfilment of the derived user requirements in User survey. The analysis will demonstrate the feasibility of applying a systematic design technique and procedures for translating the "voice" into the design and technical specifications of a robotic safety system used for TOMI Robot grass cutting such as fig 4, A laptop holder was fitted; a folded $3 \mathrm{~mm}$ flat mental and $6 \mathrm{~mm}$ wood as the central platform were comprised. It provides enough carrying capacity for putting Trimble display and communicated receiver. The laptop holder also covers the steering track rods for added user safety.

To develop safety systems for supporting operations and task planning for the smart TOMI robot working in the field, it would include elements from field management, logistics and coordination, route planning and mission optimization. The basis for the developed tools includes a comprehensive core-task analysis detailing the precise work-flow of the autonomous harvesting process. However, Mechanical Properties and Electronic systems are greatly influence on the safety system about TOMI robot. The autonomous safety system implement 
carriers would navigate in a deterministic manner within the Autonomous Hectares, based on a predefined route plan with embedded actions, developed and supplied. The visualization and verification of the predetermined rout plan will be simulated or visualized in real time with software Architecture for Agricultural TOMI Robot ${ }^{[14]}$. To realize the aim, the safety system can accurate accepting date sensors information automatically from different devises sensors about static or moving obstacles, and static and dynamic testing were carried out in the field. The safety system has been collected the data such as mainly technical parameters when meeting with urgent accident, fuel consumption and GPS data, through the sensors and computer and would have been all the optical and economical details for cutting speed ratio, the reel speed ratio, control the time of stop when meeting special circumstance and the fields working technology data. The Trimble ${ }^{\circledR}$ and FmX ${ }^{\circledR}{ }^{[15]}$ integrated display is an advanced, full-featured guidance display for the TOMI's precision farming applications. Connect with Trimble's proven precision agriculture technology and tools in TOMI's harvesting operations to support critical processes and decisions throughout the entire harvesting cycle-ultimately leading to improved cutting quality and best services for human being, lower input costs, and increased profitability.

GPS-TRIMIBLE was used for safety systems of TOMI Robot. It is used in TOMI to carry out high accuracy levels and functionality, including: field definition and mapping; Feature mapping, Create prescription maps for variable rate application of cutting grass with high quality and effectively; Guidance to predefined cutting route, logging of coverage data ${ }^{[16-17]}$; Variable rate control; To enhance the system's controlling flexibility and extensibility and help farmers more economical in their tasks. The details would be analyzed and used to benefit farm management decisions and improve efficiency. The safety system monitoring every movement has been made. Using the Trimble to TOMI, Harvest time can bring great benefit as tangible results are seen from the year's hard work ${ }^{[20]}$. With the harvest solution, precisely gather, map, monitor, and evaluate the grass yield can be made. It is benefit from precision guidance systems to accurately steer combine and follow rows - from small, irregular-shaped fields to large, open fields. Using Trimble row guidance to keep TOMI's cutter centered on rows - even in hardto-navigate areas such as down apple trees is carried out to new TOMI Robot. Grassland information as cutting and wirelessly were transferred the data,

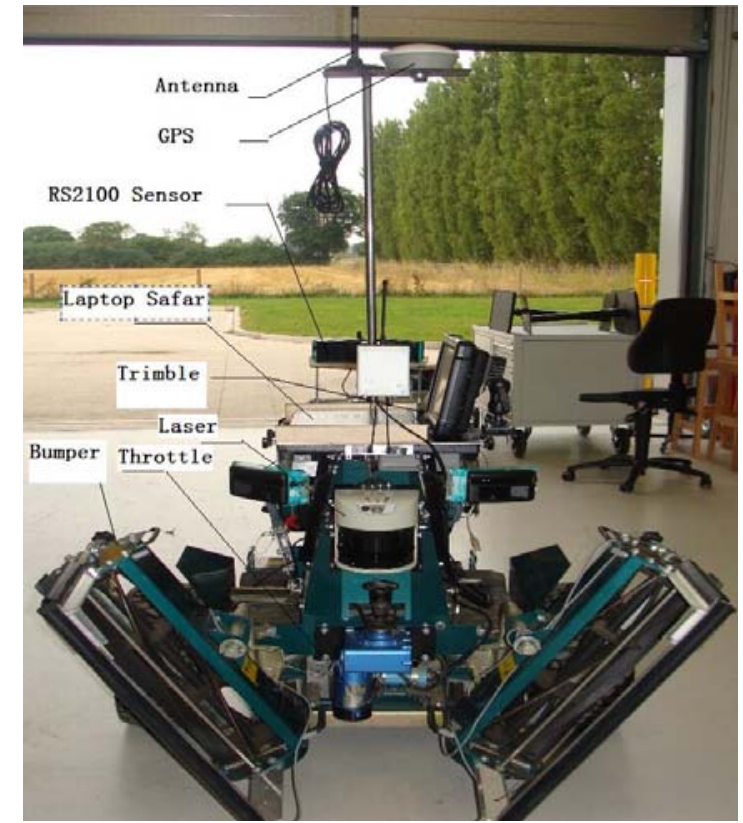

Figure4. Design and Build new safety system about TOMI

Gathering the data between fields and the office or between harvesters in the field, analysis forage yields and moisture performance of different forages in grassland. Let Trimble navigation and identify problem areas, create prescription grassland maps, and determine what best route is planning for TOMI's cutting. Leading software to use the information gathered during the growing season to make smart TOMI management decisions.

\subsection{Build new safety system and fit to TOMI robot;}

Based on the original installation of TOMI Vehicle, testing TOMI's safety system dates and following on current safety regulations, a new safety system and fit to TOMI robot have been built such as fig4. The method of FTA was used to built and fit TOMI robot's new safety systems and behaviours which have been designed. The hazard and risk analysis are detailed, and the safety problems between TOMI robot's and human a and the other animals, also the safety problems of IRM itself were deeply researched. Two Back RS2100 distance sensors with which $2 \times 11$ emitter elements scanning range of $176^{\circ}$ degrees were set at the back Torso of TOMI Robot with arranged flexible and adjustable long bolts, while the LED emitter set themselves apart through large light spot, the solutions to the safety problems of the TOMI robot were promoted, designing the wireless communication module based on ArduinoMega, LCD, LED, Ethernet and Mux shield, etc. After all, the new safety system of TOMI robot was integrated, According to the practical situation, TOMI's safety systems technological model were followed the fig 2 and fig3. The 
technology route about smart TOMI Robot was carried out and included the following stages such as fig3 and fig4、 fig4.1:

1. Route Plan of the TOMI'S Safety system;

2. Lidar Navigation (for obstacle Avoidance; Brackets-GNSS, Laser Scanner such as SICK ;)

3. Ultrasonic Ring (Traffic light system; GREEN Sensors; E-stop);

4. DEFOX Mable impact (Bumpers);

5. Big Red Buttons;

6. Dead man handle (wireless controller, radio);

7. Small +Slow +Light

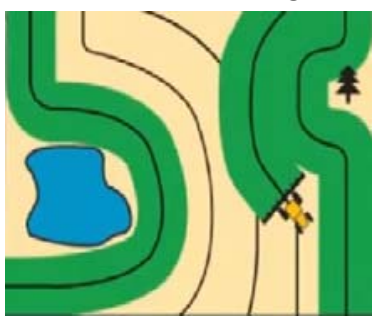

A. random

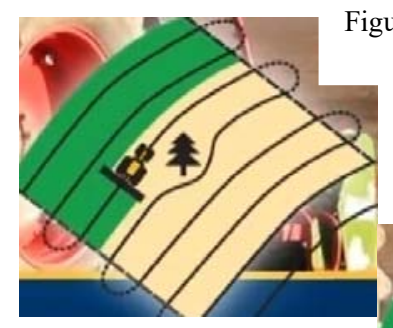

B. zigzag

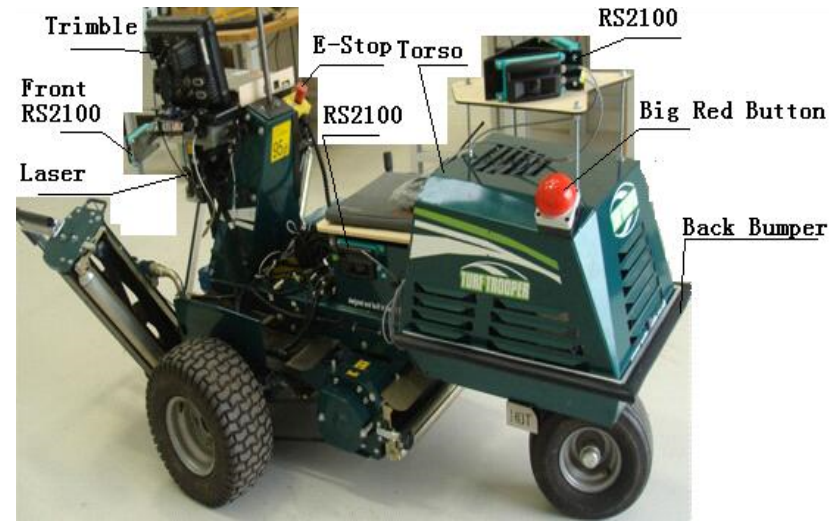

Figure4.1. Design and Build Six RS2100 Distance sensors for safety system about TOMI Robot

field, there is no particular route plan and TOMI's rout plan decisions were made on a 'case by case' basis. Path planning for rectangular fields was rather

Fig5.The types of route plans to TOMI Robot

\subsection{Route Plan of the TOMI'S Safety system}

The first stage of TOMI Robot's safety system is plan the properly path, field research for knowing the field circumstance is necessary for detecting suitable working path such as fig8. There are four types of route plans A. random, B. zigzag, C. Circle, D composites type, the type of A may be classified for TOMI'S Robot using for terrace such as fig5. For more safety and higher efficiency, as a TOMI robot, it has only three wheels, Cutting width is $1500 \mathrm{~mm}$ and Turning Radius is $686 \mathrm{~mm}$, the route plan of the TOMI'S Safety system can be planned and optimized by the practical complex conditions in the

\section{Overview of research on safety System TOMI robot.}

Technology of TOMI robot's safety system was reviewed and puts forward analysis method for the new safety systems, including safety analysis, risk assessment, risk analysis, and preventive maintenance, etc. Fault tree analysis method was carried to evaluate and analysis the TOMI robot. A deductive method for safety analysis and integration was always used such as Failure Modes and Effects Analysis (FMEA) and Fault Tree Analysis (FTA) which identify Potential unit errors resulting in hazards. This method uses well-known analysis techniques for computer control systems and includes the use of a formal language to combine safety analysis with the development process. Each

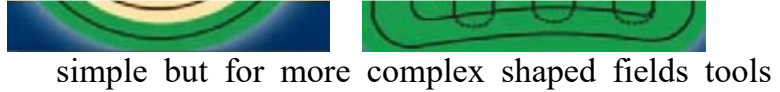
were needed. GPS developments also enable that more difficult solutions can be realized in practice. Using software to divides the complex shaped field into convex subfields is aimed to planning accurate path navigating the ROBOT working by accurate programs, for more complex fields such as fig5; it was divided in two or more subfields. Optimization techniques by TOMI's program are used to find the most optimal path. Path planning was based on that all of the working and turning area have to be covered, starting in the input area and ending in the output area.

step of the analysis was presented with a case study of a robot for safety and effectively working. Using the method of FTA analyze the potential fail factors of the intelligent TOMI robot. Safety engineering was based on product performance and cost as constraint conditions. Basing on safety of others, safety to crop and safety to itself, with professional knowledge and the method of system engineering, TOMI robot's safety system would be evaluate to the safety systems of products, improve and guarantee the safety of products, so that TOMI robot has the best safety degree of professional engineering.

The development of autonomous TOMIs in agriculture has experienced an increased interest. This development has led a few researchers to start developing more rational and adaptable TOMIs based on a behavioural approach. Simon Blackmore 
has argued that autonomous TOMI Robot for agricultural operations should behave sensibly in natural environment and have associated behaviours to deal with unknown conditions in the field ${ }^{[16-19]}$.

\subsection{The Development of safety system about TOMI'S Control Systems}

Two Control systems with hardware system and software system were designed and manufactured for TOMI's safety systems such as figure 3、 figure 4、 figure 4.1 、 figure 6 and figure 7, respectively. The hardware safety system is consisted of speeding system、steering system and functional components such as Throttle 、 Brakes and switches in fig3 、 fig4、 figure 4.1 and figure 7; the software system was consisted of all of safety systems programs such as Distance sensors and bumpers、 big red button and Dead man Handle, GPS systems and Trimble autonomously and remotely via a human operator. Aimed to Cutting forage as harvester Robot, an accurate navigation systems such as GPS and Trimble systems would be required in order to precisely detect fields. Development the safety control systems of TOMI Robot with software Architecture for the Agricultural Robot which is a guidance system specifically designed for agricultural harvester using GPS systems and Trimble systems connected with Safar network. This system is less accurate but provides an Advanced and inexpensive compact solution with the functionality required for testing and proof of concept, it is full-featured guidance display for all precision applications GPS-Trimble Agriculture Robot.

TOMI's subsystems were constructed using each associated components, including the hardware system, software systems and etc. This enabled to

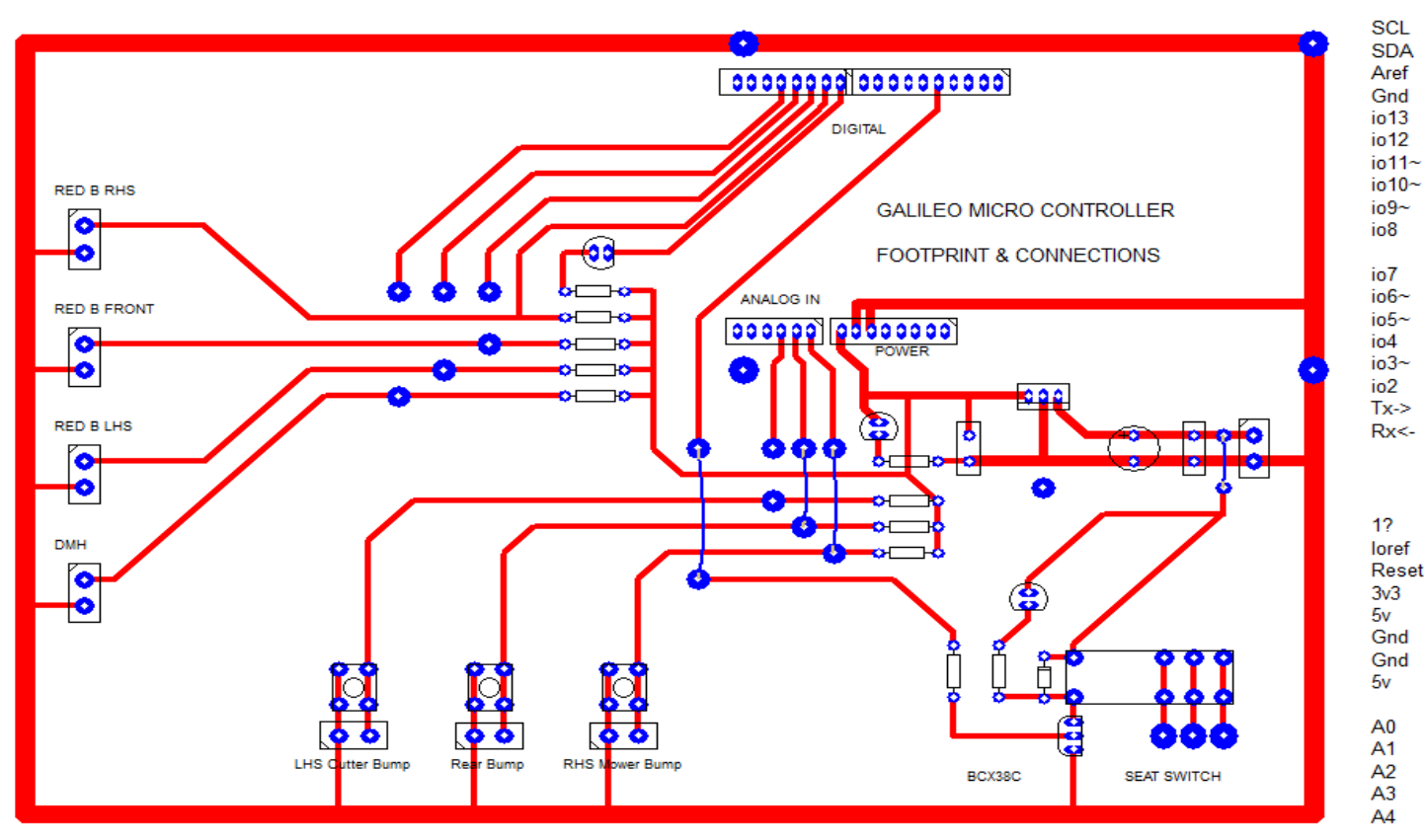

SAFETY ECU

I'IgUie U. IUIVII S LLLC INUIVIL UUIVINUL NAILI I S I ILIVI VLINIUIV L.I

fully test every

systems working control safety systems such as figure 3 and from figure 6 to figure 9. The control safety systems of new TOMI Robot are designed and developing in recent years. The detail advanced sensors technology diagrams such as how much this type of sensors and pins were designed and used as following fig 7 .

TOMI Robot can be controlled both the test of TOMI's safety system successfully navigated following several pre-planned routes with a high level of accuracy and repeatability.

The speeding and steering hardware systems were primarily designed for TOMI's safety control systems and as such the output provided were not subsystem and to assess the TOMI Robot's suitability. Test results were promising but did highlight the importance for accurately calibrating the GNSS system and correctly tuning the Proportional Integral Derivative control systems for the steering and speeding outputs. With the system correctly tuned,

was

ideally suited for harvester's application straight out of the box. The output are consists of a throttle control and a steering control signal. This output could be directly connected to the steering and speed hardware on the automatically harvester, but 
there would be no provision for object avoidance or safety systems. In order to overcome this output from the Ethernet has been connected to an Arduino
Mega board; GPS system and Trimble system have been connected to computer allowing the use of additional navigation systems such as fig3.

\section{TOMMI RESEARCH VEHICLE.}

SAFETY system.
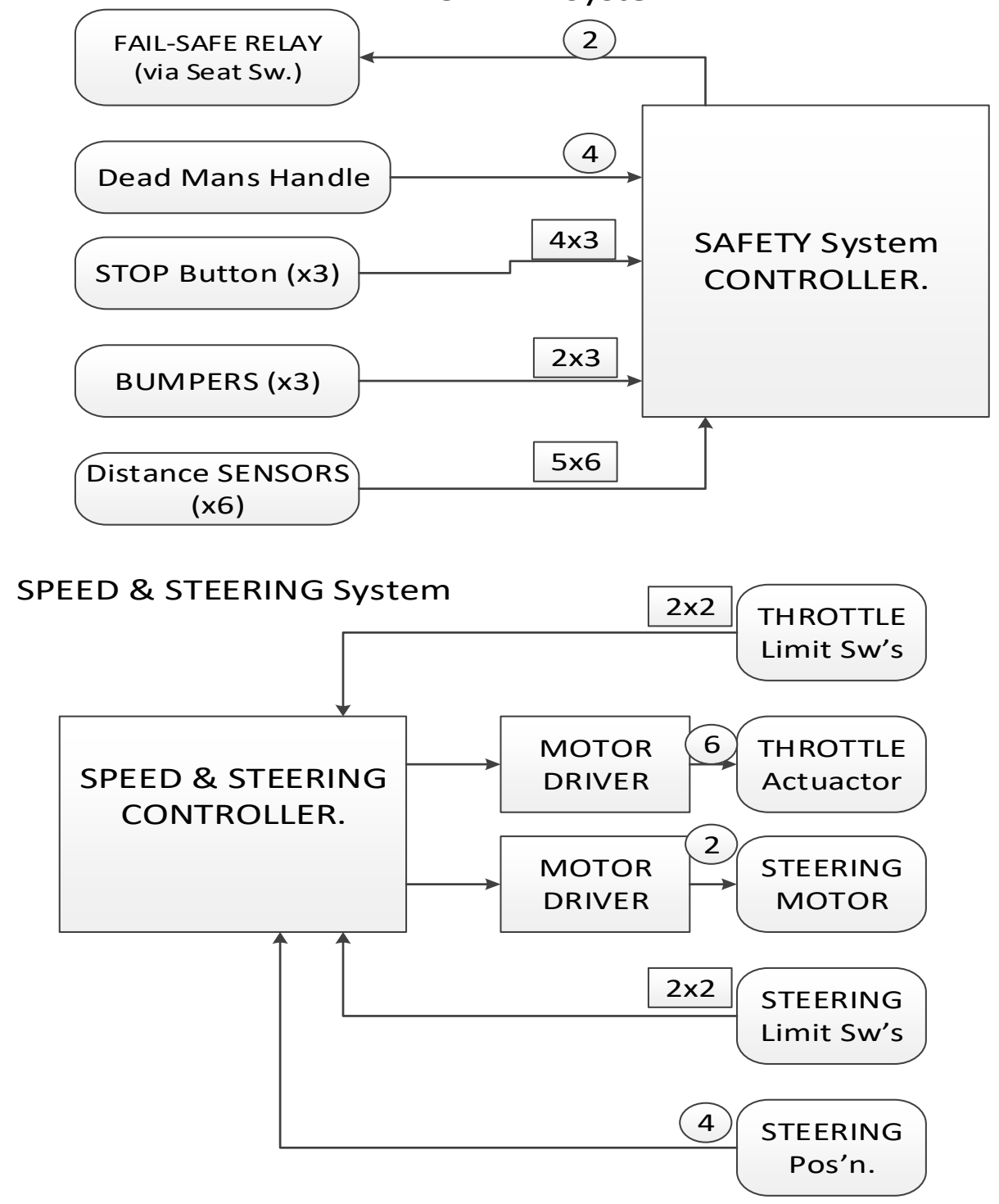

Figure7. TOMI Safety Systems Connectors pins Diagrams 


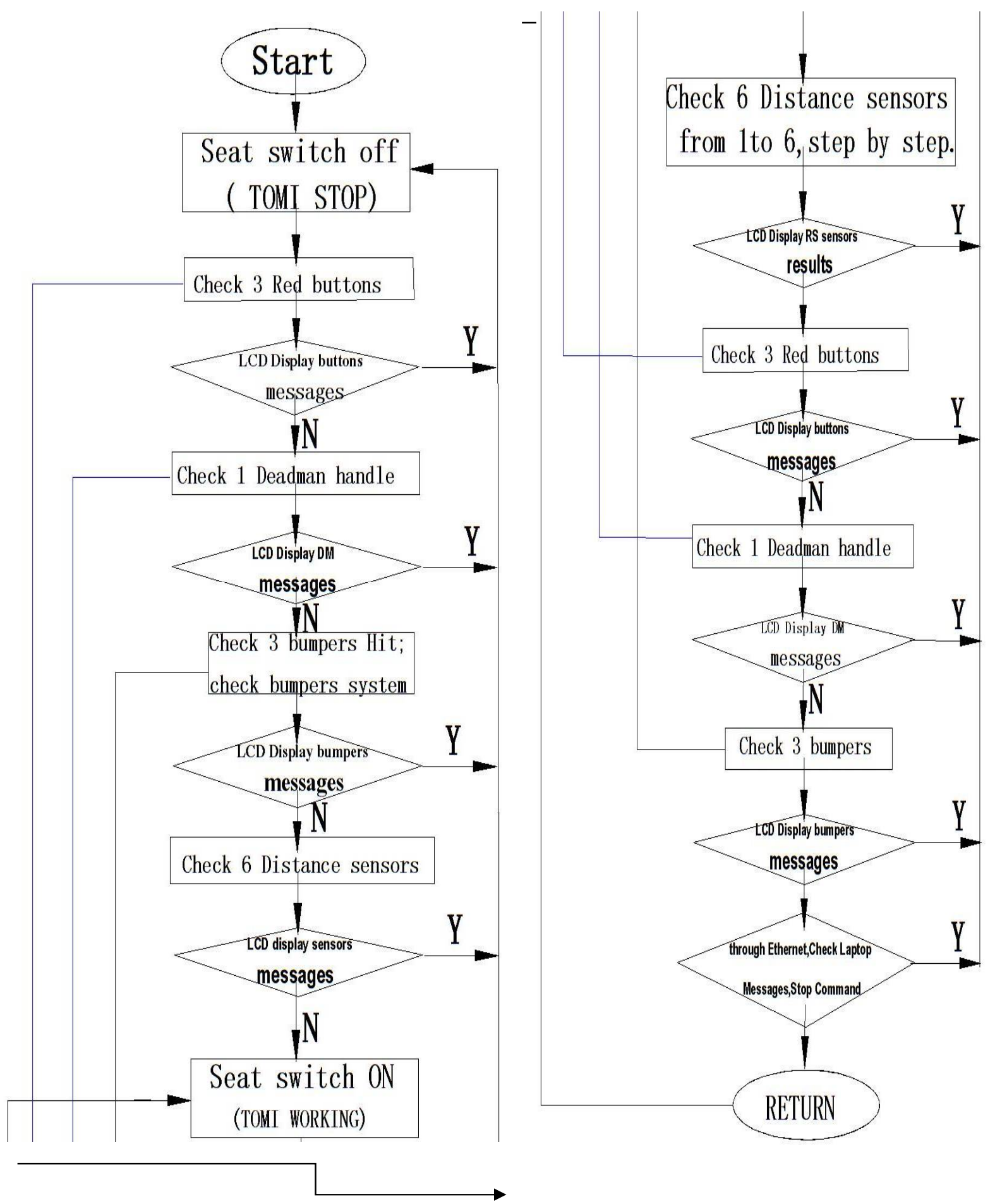

Fig9. THE SOFTWARE FLOWCHART OF TOMI SAFETY SYSTEM

\subsection{The Development of Safety systems about TOMI Robot}

As TOMI Robot is a harvester Robot, it is intended to be working in various terrace fields and harvesting various forages, it should be able to detect surrounding and deal with that. As well as often having irregular shapes may be presented a wide range of obstacles such as trees v tractors and telegraph poles Such as Figure8. 


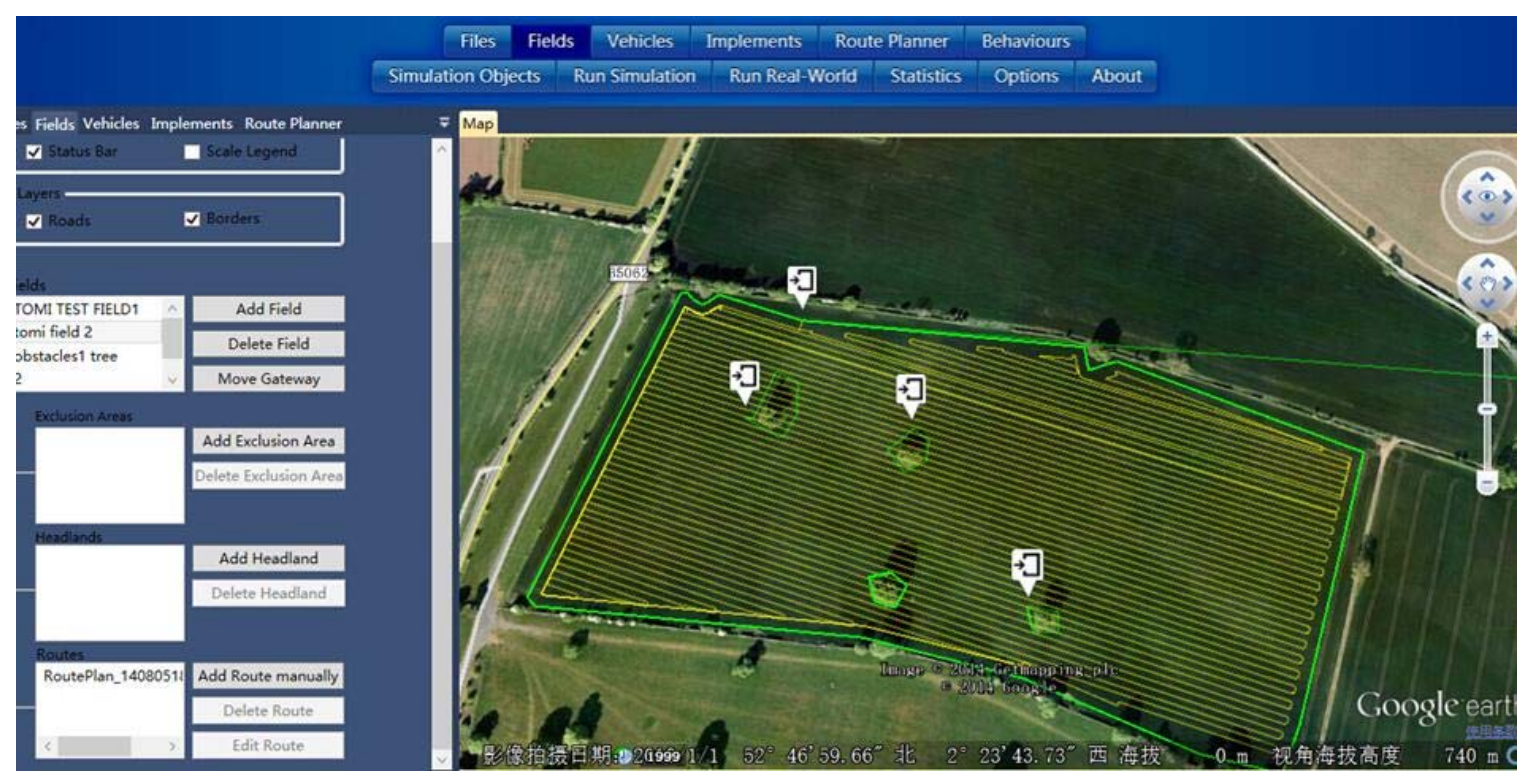

Figure8. Detect obstacles to the new Safety systems of TOMI Robot (Adapted from: authors and http://www.Web Farming)

It is possible to plan route on the guidance software programs on TOMI robot to take into account static objects such as trees, telegraph poles and dynamic objects such as tractors、 persons, etc. Various sensors were researched for objects detection including infrared, ultrasonic and laser range finders, RS2100 distance sensors is belong to multifunction scanner with wide-beam emitters and can separate various forages from the surrounding different other breeds of forages, it usually was selected for additional navigation systems just like GPS systems and Trimble system using for harvesting in application. One of the main advantages is the increased level of accuracy afforded when detecting fields. Operating results in much less overlap which increases the efficiency of the operations dramatically have been carried out. Along with this, removing out operator from the TOMI robot means that the Smart TOMI Robot can working in harvesting periods for 24 hours a day because of reliable hydraulic systems.

Basic overviews of the Smart TOMI Robot and the main components have been studied in Harper Adams University. Although a LMS511 type of Laser scanner is $190^{\circ}$ degree scanning unit, it was accurately decided to run away when make up to obstacles in front of new TOMI robot. It allows the Laser scanner to detect various obstacles. The control systems are housed in an Arduino Mega, Ethernet and Max shield rated box in the centre of the harvester Robot in order to keep them more safety. Similarly the Pixhawk and GNSS systems were housed in a similar Plastic Box.

\section{Testing the Programs of TOMI's}

\section{new software Safety Systems}

New TOMI's safety control systems were consisted of two safety systems, AS times and location were limited, The first part of safety systems were checked about the software safety systems of SWITCHES、BIG RED BUTTON、DEADMAN HANDLE 、BUMPERS、DISTANCE SENSORS and SEAT SWITCH such as from fig6 to fig9. The TOMI software safety systems were downloaded from Laptop by WIFI to Arduino Mega. The software programs of TOMI'S safety systems have more multifunctions with complex and interfered because of Agricultural TOMI Robot. The Fault Code Structure of the software was used the 'Fault Code "-> "word" (16 bits) split into 4 nibbles and Fault Code starts at 0xFFFF (all faults active); Function of System Check clears from 1's to zero's; when fault code all zero's vehicle was in operating mode and any change to Fault Code would activate function Fail Safe. Top line on the LCD display the information, bottom line displays the fault codes. The program of TOMI's safety control systems was set up function and executed with circle programs. The power was connected and switches were turned on, and close up the IR Sensors TX, a sign on message at 9600 baud would showed out, over first few seconds; before up shift to $115 \mathrm{k} 2$ baud rate and request first distance frame, the checking System could include TX CR/LF and check R2100> response. Testing periods need to notes: loop duration time measurements; response to confirm processor time slots, implement Serial commas timeout and implement received frame header detecting such as LMS511 type of Laser scanner 
ensures precise guidance and obstacle avoidance with high response speeds for harvesting processes.

Firstly, the $12 \mathrm{~V}$ power button and LCD1602's button were turned on, than, the system of Rear Left Hand Switch, Rear Right Hand Switch and Front Switch were checked respectively, if all of them ok, the LCD1602 would display "Fault Code =0x0100"; "Fault Code =0x0200" and"Fault Code =0x0400", else showing "Fault Code $=0 \mathrm{x} 0$ " and immediately,

\section{CONCLUSION}

1) Based on testing results, the significant difference distances were clearly showed in the same working circumstance such as table 1 , the nearest distances between the left clutch pedal and right brake pedal of the mechanical structure is only $322 \mathrm{~mm}$, the original TOMI's mechanical structure working in the field was led to unsafely, as the result of that, precision structure and control safety systems of developing the new TOMI's robot were built up and development.

2) Designing Six Distance sensors on new safety control systems to TOMI Robot made it scanning up to $360^{\circ}$ degree in 3D direction and effective working distance was about to $10 \mathrm{~m}$, Big Red buttons, Dead man Handle and Bumpers were set on suitable places to make TOMI more safety and effective.

3) Building the new safety systems of TOMI robot can be controlled both autonomously and remotely control. In order to achieve reasonably navigational system, GPS system and Trimble system combined with WIFI-Antenna with which accurate and advanced intelligence safety control components were required to precisely navigate various grassland、crops fields or terrace fields, but it is expensive to develop the Forage harvester the Seat Switch would turn off. Checking R2100 distance sensors step by step, if it is ok, it shows "SYS CHK OK", next, checking the Dead man handle with the same method of Switches, then according to Bumpers value, TOMI's bumpers system may be checked when connected with $8 \mathrm{~K}$ resistances. If bumpers are touched with more than 2.1 2.5N, the LCD1602 shows "bumpers ok", then continue to check distance sensor and response.

ROBOT using in mountain area. Aimed to harvesting nutritious Alfalfa named as "Forages Queen" globally, the agriculture harvesting Robot named TONNI with accurate guidance system would be development, it is full-featured guidance display for all precision applications GPS and Trimble Agriculture. It will be continued to develop more practical and safety systems of TONNI robot using for northwest mountain area.

\section{ACKNOWLEDGEMENT}

The author would like to thanks for supervisor professor Simon Blackmore and all of the staffs in Engineering College at Harper Adams University; the research has been carried out at national precision Agriculture Engineering innovative Centre in UK. It was gratefully appreciated the research and acknowledge financial supporting from the CHINA SCHOLARSHIP COUNCIL (CAC), The paper would be funded by Special Fund for Agro-scientific Research in the Public Interest(201203024) and National Natural Science Foundation of China (51565002); Research and Development of the main Project in Gansu (17YFINA061) and The First Fuxi outstanding talent candidates project in Gansu Agricultural University 


\section{Reference:}

1 Chrysler. Potential failure mode and effects Analysis FMEA (Fourth Edition). Ford Motor Company, General Motors Corporation (2008).

2 Kung L, Stough E C, McDonell E E, etc. The effect of wide swathing on wilting times and nutritive value of alfalfa haylage. Journal of Dairy Science, 93 (4):1770-1773(2010)

3 Nehmzow, U. Mobile Robotics, a Practical Introduction. 2nd Edition. Springer. ISBN 1-85233-726-5 2nd edition Springer-Verlag London Berlin Heidelberg (2003).

4 Robertson, D. Linehan, D. Failure Mode and Effects Analysis. Society of Motor Manufacturers and Traders Limited. SMMT by Findlay Publications Ltd, Horton Kirby, Kent DA4 9LL (2003).

5 Morgan, S. Programming Microsoft Robotics Studio. Microsoft press A Division of Microsoft Corporation one Microsoft way Redmond, Washington 98052-6399(2008).

6 PREDKO. M. 123 Robotics Experiments for the Evil Genius. McGraw-Hill New York Chicago ,San Francisco Lisbon London Madrid Mexico City Milan NEW Delhi San Juan Seoul Singapore Sydney Toronto.ISBN0071413588(2003).

7 E.J. Van Herten D. Goense C.Lokhorst. Precision agriculture'09. Wageningen Academic Publishers. Papers presented at the 7th European Conference on Precision Agriculture Wageningen, the Netherlands 6-8 July 2009.

8 Rodrigo S. Jamisola, Jr. \&Anthony A. A Path Planning Strategy for Kinematically Redundant Manipulators Anticipating Joint Failures in the Presence of Obstacles. Proceedings of the 2003 IEEE/RSJIntl. Conference on Intelligent Robots and Systems as Vegas, Nevada · October 2003.

9 3D Robotics, 2014. 3DR Pixhawk. Available from on- line. https://store.3drobotics.com/products/3drpixhawk (2014). Accessed 10 April 2014

10 Active Robots. 2013. Firgelli. Active Robots Limited. Available from On-Line: http://www.activerobots.com/brands/firgelli (2014). Accessed :14 May 2014

11 PC1602 Series: Available from: https://www.google.co.uk. Accessed May 2014

12 Amazons, 2014. AmazoneBonirob. Available from: http://go.amazone.de/?lang=1\&news=26 .Accessed 10 April 2014

13 Arduino, 2014. Arduino Project. Available from: http://arduino.cc/en/Main/Products .Accessed 10 April 2014

14 Chunhua Zhao, Simon Blackmore, Michael War brick, Sam Wane. Test, Analysis and Evaluation of RS2100 Distance Sensor used for Robot Grass Cutting, International conference on Mechanistic, Electronic, Industrial and Control Engineering (MEIC2015), 277-281,2015

15 Kang, S. C., Chang, W. T.. Robot Development Using Microsoft ROBOTICS Developer Studio, Taylor \& Francis Group Boca Raton London New York(2011).

16 Blackmore, B. S., Fountas, S. \& Have, H., 2004a. System Requirements For a Small Autonomous Tractor. CIGR Journal of Scientific Research and Development, July 2004, p. Manuscript PM 04001.

17 RS2100-datasheet. Available from On-Line: https://www.google.co.uk.

18 Blackmore, B. S. et al., 2004b. Development of a deterministic autonomous tractor. Beijing, CIGR International Conference(2004)

19 Dorhoutrd and D, 2013. Prospero; Robot Farmer. Available from On-line: http://dorhoutrd.com/prospero robot farmer. Accessed 10 April 2014

20 BBC. Farmers say they're struggling after a bad harvest. British Broadcasting Company. Available from OnLine: http://www.bbc.co.uk/newsbeat/19899337 (2012) 